\title{
Interesting features of a general class of higher-derivative theories of quantum gravity
}

\author{
A. Accioly, ${ }^{*}$ J. de Almeida, ${ }^{\dagger}$ G. P. de Brito, ${ }^{*}$ and W. Herdy ${ }^{\S}$ \\ Centro Brasileiro de Pesquisas Físicas (CBPF), Rua Dr Xavier Sigaud 150, Urca, \\ Rio de Janeiro CEP 22290-180, RJ, Brazil
}

(Received 5 June 2018; published 14 September 2018)

\begin{abstract}
In this work we investigate an interesting connection between the absence of Newtonian singularities in the classical nonrelativistic potential and renormalizability properties in higher-derivative models of quantum gravity. In the framework of a large class of $D$-dimensional higher-derivative models of quantum gravity, we compute the nonrelativistic potential energy associated with two pointlike masses. Investigating its behavior for small distances, we find an algebraic condition which is sufficient for the cancellation of the Newtonian singularity. We verify that the same condition is necessary to ensure power-counting renormalizability and, as a consequence, we conclude that renormalizable higher-derivative models do not exhibit the so-called Newtonian singularity. Finally, we discuss the role of ghosts in the mechanism for the cancellation of Newtonian singularities.
\end{abstract}

DOI: 10.1103/PhysRevD.98.064029

\section{INTRODUCTION}

The quest for a quantum gravity theory is still one of the most important problems of theoretical physics. As is well known, the biggest challenge in the construction of a quantum theory for the gravitational interaction is the lack of experimental evidences concerning gravity at the microscopic level. Despite that, however, there exist several approaches to quantum gravity which were proposed in the past few decades; for instance, string theory, loop quantum gravity, causal dynamical triangulations, causal sets and induced quantum gravity $[1,2]$. Nevertheless, none of the aforementioned theories can be considered a complete quantum gravity theory up to now.

At the classical level, the gravitational interaction is very well described in terms of Einstein's general relativity (GR), which is confirmed for the excellent concordance between its theoretical predictions and the available experimental tests (e.g., solar system tests, cosmological observations and the recent discovery of gravitational waves). Therefore, a natural path for the construction of a quantum

\footnotetext{
accioly@cbpf.br

josejr@cbpf.br

gpbrito@cbpf.br

\$wallacew@cbpf.br
}

Published by the American Physical Society under the terms of the Creative Commons Attribution 4.0 International license. Further distribution of this work must maintain attribution to the author(s) and the published article's title, journal citation, and DOI. Funded by SCOAP ${ }^{3}$. theory of gravity seems, at least at first sight, the quantization of GR.

Now, keeping in mind that the fundamental field concerning GR is the spacetime metric, a possible way to perform its quantization entails a functional integration over all metric fluctuations around some vacuum configuration [1,3]. For instance, we may consider the metric splitting $g_{\mu \nu}=\eta_{\mu \nu}+\kappa h_{\mu \nu}$ and perform afterward the path integral quantization of the fluctuation field $h_{\mu \nu}$. In this approach we can derive at the tree level the Feynman rules of the theory and therefore apply the standard techniques of perturbative quantum field theory. An interesting result that can be obtained via the semiclassical approach is the gravitational light bending [4]. Nevertheless, computations at the loop level are problematic. In this case, the UV divergences are not treatable by means of perturbative renormalization and, as a consequence, the theory is UV incomplete.

A possible way out of the UV divergences problem is the introduction of higher-derivative terms in the usual EinsteinHibert action. ${ }^{1}$ In fact, these higher-derivative terms improve the behavior of the tree-level propagator in such a way that it compensates the "nasty" UV behavior of the vertices containing derivative couplings. In a seminal paper [6], Stelle investigated thoroughly the renormalizability of

\footnotetext{
${ }^{1}$ An alternative route to deal with that problem is the so-called Asymptotic Safety program [5]. On the other hand, within the context of Asymptotic Safe Quantum Gravity the problem of UV divergences may be carried out via the use of nonperturbative renormalization. In this paper, we shall restrict ourselves to perturbative techniques.
} 
higher-derivative theories of quantum gravity and came to the conclusion that a fourth-derivative theory described by an action containing curvature-squared terms is renormalizable (in four dimensions) to all orders of perturbation theory. However, there is a price to be paid for attaining renormalizability: the spectra of the theory exhibits a massive ghostlike particle which can cause unitarity violation. In addition, from the classical point of view, higher derivatives may lead to Ostrogradsky's instabilities. Unfortunately, nonunitarity is a problem as undesirable as nonrenormalizability. Therefore, we have to find a way to circumvent it if we want to follow the route of higher-derivative theories of quantum gravity. ${ }^{2}$

In the past few decades many efforts have been made to reconcile unitarity and renormalizability of higherderivative theories. For instance, it was verified that if we allow terms with six or higher derivatives in the action, then the theory becomes superrenormalizable [8-10] and it is possible to find some region in the parameter space where all the nontrivial poles of the tree-level propagator are complex and, therefore, the theory may be formulated as unitary in the Lee-Wick sense [11,12]. We remark, however, that the presence of complex poles may lead to problems with causality [13]. Another possibility arises if we allow nonlocal terms in the action [14-16]. In this case it is possible to choose a form factor such that the only pole of the propagator corresponds to the usual physical graviton and, as a consequence, we may escape from the unitarity violation. Besides, a typical nonlocal term improves the tree-level propagator in such a way that the theory becomes superrenormalizable or even UV finite. ${ }^{3}$ In the past few years the study of nonlocal theories of quantum gravity has been applied to several physical situations, e.g., cosmological scenarios and astrophysical properties $[18,19]$, high energy scatterings [20] and so on.

If we restrict ourselves to local gravitational theories, however, renormalizability and unitarity cannot be simultaneously achieved. A conjecture first proposed by Stelle [6] that renormalizable higher-order gravity models are endowed with a classical potential lacking a singularity at the origin allows us to look at this incompatibility through a different lens. Since a unitary system is correlated to a singular potential at the origin [21], while a renormalizable model is related to a potential finite at the origin, this conjecture has as a consequence the impossibility of having higher order gravitational theories that are simultaneously unitary and renormalizable.

\footnotetext{
${ }^{2}$ Remarkably, a Euclidean lattice formulation of the fourthderivative quantum gravity points out that unitarity may be restored at nonperturbative level [7].

${ }^{3}$ It is important to emphasize that there are some classes of nonlocal theories where both renormalizability and tree-level unitarity are respected; however, after quantum corrections are introduced it is possible that they become nonunitary [17].
}

Recently, some authors have addressed the problem of verifying this hypothesis for several models. It has been verified in $D$-dimensions for fourth- and sixth-derivative derivative gravity [22,23] and also for scale invariant gravity [24]. The converse of this hypothesis was shown to be false, in other words, that a higher-order gravitational model which has a finite classical potential at the origin is not necessarily renormalizable [25].

In this paper we intend to probe this conjecture for a general class of $D$-dimensional higher-derivative gravity theories. In this vein, we compute a general expression for the interparticle potential energy and analyze its behavior for small distances. A sufficient condition for the regularity of the potential at small distances is then found and, as we shall see, this condition turns out to be automatically satisfied by power-counting renormalizable theories. For the sake of completeness, we shall discuss the tree-level unitarity of this general class and verify that the theories which are ghost-free turn out to be power-counting nonrenormalizable. In addition, the role of ghostlike particles on the cancellation of the Newtonian singularity is discussed as well $[25,26]$.

This paper is organized as follows: In Sec. II, we present a general class of higher-derivative gravity models and investigate some of its properties. In Sec. III we compute a general expression for the interparticle potential and discuss its behavior for small distances. In Sec. IV, we study the UV behavior of the system and its renormalizability properties. In Sec. V, we analyze the particle spectra of the theory as well as its implications for tree-level unitarity. In Sec. VI, we summarize our findings and probe the conjecture that the absence of Newtonian singularities is a necessary condition for these theories to be renormalizable. Finally, in Sec. VII we present our conclusions.

Throughout this paper we use the conventions $c=\hbar=1$, $\eta_{\mu \nu}=\operatorname{diag}(+,-, \cdots,-), R_{\nu \alpha \beta}^{\mu}=\partial_{\alpha} \Gamma_{\nu \beta}^{\mu}+\Gamma_{\alpha \lambda}^{\mu} \Gamma_{\nu \beta}^{\lambda}-(\alpha \leftrightarrow \beta)$, $R_{\mu \nu}=R^{\beta}{ }_{\mu \nu \beta}$ and $R=g^{\mu \nu} R_{\mu \nu}$.

\section{GENERAL CLASS OF HIGHER-DERIVATIVE GRAVITY MODELS}

Let us start considering a general class of higherderivative gravity models. Although the gravitational action has an infinite number of terms that are compatible with the symmetries under general coordinate transformations, we shall only consider the quadratic sector of the action. ${ }^{4}$ In this spirit, the most general $D$-dimensional $(D \geq 3)$ action is given by

\footnotetext{
${ }^{4}$ Naturally the discussion of renormalizability takes into account nonquadratic terms. However, the specific form of these contributions will not be relevant for our purposes.
} 


$$
\begin{aligned}
S\left[g_{\mu \nu}\right]= & \int d^{D} x \sqrt{|g|}\left(\frac{2 \sigma}{\kappa^{2}} R+\frac{1}{2 \kappa^{2}} R F_{1}(\square) R\right. \\
& \left.+\frac{1}{2 \kappa^{2}} R_{\mu \nu} F_{2}(\square) R^{\mu \nu}\right),
\end{aligned}
$$

where $\kappa^{2}=32 \pi G=32 \pi M_{\mathrm{Pl}}^{2-D}$ is the gravitational coupling constant, $\sigma$ is a parameter that can be taken to be either +1 or -1 and $F_{1}(\square)$ and $F_{2}(\square)$ are functions of the covariant d'Alembertian operator $\left(\square=g^{\mu \nu} \nabla_{\mu} \nabla_{\nu}\right)$. Moreover, we shall assume that the functions $F_{1}(\square)$ and $F_{2}(\square)$, the so-called form factors, have a finite polynomial representation.

At this point some comments regarding the action above are in order:

(i) As it was mentioned before, our goal is to consider the most general contribution for the quadratic sector of the gravitational action. In this sense, there are several nontrivial steps before Eq. (1). As it was argued in Refs. [14,27], the most general contribution involving quadratic curvature terms takes the following form:

$$
\int d^{D} x \sqrt{|g|} R_{\mu \nu \alpha \beta} \mathcal{D}_{\rho \lambda \sigma \gamma}^{\mu \nu \alpha \beta} R^{\rho \lambda \sigma \gamma},
$$

where $\mathcal{D}_{\rho \lambda \sigma \gamma}^{\mu \nu \alpha \beta}$ is a differential operator constructed with all possible combinations involving covariant derivatives and the spacetime metric. At first sight, this contribution can be written in terms of fourteen invariant terms [see Eq. (3) in Ref. [27]]. After some rearrangements based on the use of the antisymmetric properties of the Riemann tensor and also using the Jacobi identity, the most general curvature quadratic contribution can be recast in terms of six independent operators, namely

$$
\begin{aligned}
& \int d^{D} x \sqrt{|g|}\left(R F_{1}(\square) R+R_{\mu \nu} F_{2}(\square) R^{\mu \nu}\right. \\
& \quad+R_{\mu \nu \alpha \beta} F_{3}(\square) R^{\mu \nu \alpha \beta}+R F_{4}(\square) \nabla_{\mu} \nabla_{\nu} \nabla_{\alpha} \nabla_{\beta} R^{\mu \nu \alpha \beta} \\
& \quad+R_{\mu}^{\lambda \rho \sigma} F_{5}(\square) \nabla_{\lambda} \nabla_{\rho} \nabla_{\sigma} \nabla_{\nu} \nabla_{\alpha} \nabla_{\beta} R^{\mu \nu \alpha \beta} \\
& \left.\quad+R^{\lambda \rho \sigma \gamma} F_{6}(\square) \nabla_{\lambda} \nabla_{\rho} \nabla_{\sigma} \nabla_{\gamma} \nabla_{\mu} \nabla_{\nu} \nabla_{\alpha} \nabla_{\beta} R^{\mu \nu \alpha \beta}\right) . \quad \text { (3) }
\end{aligned}
$$

Since we are interested in considering only quadratic contributions with respect to $h_{\mu \nu}$, we can replace those covariant derivatives appearing in the last expression by ordinary derivative operators. In these conditions, the derivative operators commute and, as a consequence, those terms associated with the form factors $F_{4}(\square), F_{5}(\square)$ and $F_{6}(\square)$ vanish. It should be emphasized that some points of the above discussion rely on the assumption that we are dealing with fluctuations defined with respect to a Minkowskian background. For a discussion about higher-derivative models with (anti-)de Sitter background, we recommend $[28,29]$. Also, it is important to mention that the classical dynamics associated with this general class of quadratic curvature gravity was studied within the full nonlinear regime in Ref. [30].

(ii) Now we turn our attention to the contribution coming from the invariant term

$$
R_{\mu \nu \alpha \beta} F_{3}(\square) R^{\mu \nu \alpha \beta} .
$$

Indeed, this is a legitimate term from the point of view of spacetime symmetries and it apparently contributes to the quadratic sector. However, looking closer this is not completely true. In fact, taking into account small fluctuations around the Minkowskian background, $g_{\mu \nu}=\eta_{\mu \nu}+\kappa h_{\mu \nu}$, we arrive at the following result:

$$
\begin{aligned}
R_{\mu \nu \alpha \beta} F_{3}(\square) R^{\mu \nu \alpha \beta}= & 4 R_{\mu \nu} F_{3}(\square) R^{\mu \nu} \\
& -R F_{3}(\square) R+\partial \Omega+\mathcal{O}\left(h^{3}\right) .
\end{aligned}
$$

Since we are mainly interested in the quadratic part of the action we can discard the contribution of $R_{\mu \nu \alpha \beta} F_{3}(\square) R^{\mu \nu \alpha \beta}$ by a simple redefinition of the functions $F_{1}(\square)$ and $F_{2}(\square)$. To be precise, if we start with

$$
\begin{aligned}
S\left[g_{\mu \nu}\right]= & \int d^{D} x \sqrt{|g|}\left(\frac{2 \sigma}{\kappa^{2}} R+\frac{1}{2 \kappa^{2}} R F_{1}(\square) R\right. \\
& +\frac{1}{2 \kappa^{2}} R_{\mu \nu} F_{2}(\square) R^{\mu \nu} \\
& \left.+\frac{1}{2 \kappa^{2}} R_{\mu \nu \alpha \beta} F_{3}(\square) R^{\mu \nu \alpha \beta}\right)
\end{aligned}
$$

and then consider Eq. (5), we may rewrite the last expression as follows:

$$
\begin{aligned}
S\left[g_{\mu \nu}\right]= & \int d^{D} x \sqrt{|g|}\left[\frac{2 \sigma}{\kappa^{2}} R+\frac{1}{2 \kappa^{2}} R\left(F_{1}(\square)\right.\right. \\
& \left.-F_{3}(\square)\right) R+\frac{1}{2 \kappa^{2}} R_{\mu \nu}\left(F_{2}(\square)\right. \\
& \left.\left.+4 F_{3}(\square)\right) R^{\mu \nu}\right]+\int d^{D} x \partial \Omega,
\end{aligned}
$$

where we have removed the contribution $\mathcal{O}\left(h^{3}\right)$. Keeping in mind that $\int d^{D} x \partial \Omega=0$ with the proper boundary condition and using the following redefinitions, 


$$
\begin{aligned}
F_{1}(\square)-F_{3}(\square) & \mapsto F_{1}(\square) \quad \text { and } \\
F_{2}(\square)+4 F_{3}(\square) & \mapsto F_{2}(\square),
\end{aligned}
$$

we recover our original action (1).

(iii) As it was mentioned before, there is an infinite number of compatible terms with the symmetries of the gravitational interaction. These terms may be obtained through all possible invariant combinations of the Riemann tensor, the Ricci tensor and the Ricci scalar, e.g., $R^{3}, R_{\mu \nu} R^{\mu \nu} R, R_{\mu \nu \alpha \beta} R^{\mu \alpha} R^{\nu \beta}, R^{4}$, $\left(R_{\mu \nu} R^{\mu \nu}\right)^{2}$ and so on. However, it is not difficult to see that the aforementioned contributions bring only terms of order $\mathcal{O}\left(h^{3}\right)$, which are not relevant to the quadratic part of the action.

(iv) Finally, we have introduced the constant parameter $\sigma$ in order to explore some interesting features related to the unitarity of three-dimensional higherderivative models. In addition, without loss of generality we will take this parameter to be either $\sigma=+1$ or $\sigma=-1$.

\section{NONRELATIVISTIC POTENTIAL ENERGY}

In order to analyze the conjecture that relates renormalizability to the cancellation of Newtonian singularities, let us compute the interparticle potential energy for the general higher-derivative gravity theory given by (1) and investigate its behavior for small distances. For this purpose we shall employ the prescription presented by Accioly et al., which is based on the path integral formulation of quantum field theory [31]. This prescription states that, in order to determine the potential energy of gravitational models, we need only to compute

$$
E_{D}(r)=\left.\frac{\kappa^{2}}{4} \frac{M_{1} M_{2}}{(2 \pi)^{D-1}} \int d^{D-1} \mathbf{k} e^{\mathbf{k} \cdot \mathbf{r}} P_{00,00}(k)\right|_{k_{0}=0},
$$

where $P_{00,00}$ is the $\mu=\nu=\alpha=\beta=0$ component of the modified propagator $P_{\mu \nu, \alpha \beta}=D_{\mu \nu, \alpha \beta}-D_{\mu \nu, \alpha \beta}^{\perp}$, with $D_{\mu \nu, \alpha \beta}^{\perp}$ being the contribution to the propagator that is orthogonal to the energy-momentum tensor, while, as before, $\kappa^{2}=32 \pi G$.

After a standard procedure [32], the free propagator (in momentum space) associated with the general higherderivative gravity (1) may be cast as

$$
\begin{aligned}
\mathcal{D}_{\mu \nu, \alpha \beta}(k)= & \frac{1}{\sigma k^{2} Q_{2}\left(k^{2}\right)} P_{\mu \nu, \alpha \beta}^{(2)}-\frac{1}{(D-2)} \frac{1}{\sigma k^{2} Q_{0}\left(k^{2}\right)} P_{\mu \nu, \alpha \beta}^{(0-s)} \\
& +\frac{2 \lambda}{k^{2}} P_{\mu \nu, \alpha \beta}^{(1)}+\left(\frac{4 \lambda}{k^{2}}-\frac{(D-1)}{\sigma(D-2) k^{2} Q_{0}\left(k^{2}\right)}\right) P_{\mu \nu, \alpha \beta}^{(0-w)} \\
& -\frac{\sqrt{D-1}}{\sigma(D-2) k^{2} Q_{0}\left(k^{2}\right)}\left(P_{\mu \nu, \alpha \beta}^{(0-s w)}+P_{\mu \nu, \alpha \beta}^{(0-w s)}\right),
\end{aligned}
$$

where $\left\{P^{(2)}, \ldots, P^{(0-w s)}\right\}$ denotes the set of Barnes-Rivers operators, $\lambda$ is the gauge fixing parameter ${ }^{5}$ and we have defined

$$
Q_{2}\left(k^{2}\right)=1+\frac{1}{4 \sigma} k^{2} F_{2}\left(-k^{2}\right),
$$

and

$Q_{0}\left(k^{2}\right)=1-\frac{k^{2}}{\sigma(D-2)}\left((D-1) F_{1}\left(-k^{2}\right)+\frac{D}{4} F_{2}\left(-k^{2}\right)\right)$.

It is important to emphasize that although we are dealing with polynomial form factors (i.e., local theories), the propagator above reported is also valid for the case of nonlocal form factors and it can be compared with those results presented in $[27,33]$.

Now, since we have already determined the propagator in (10), it is straightforward to see that

$$
P_{00,00}(k)=\frac{D-2}{D-1} \frac{1}{\sigma k^{2} Q_{2}\left(k^{2}\right)}-\frac{1}{(D-1)(D-2)} \frac{1}{\sigma k^{2} Q_{0}\left(k^{2}\right)} .
$$

As it was previously mentioned $F_{1}(\square)$ and $F_{2}(\square)$ are polynomial functions of the d'Alembertian operator, namely

$F_{1}(\square)=\sum_{n=0}^{p} \alpha_{n}(-\square)^{n} \quad$ and $\quad F_{2}(\square)=\sum_{n=0}^{q} \beta_{n}(-\square)^{n}$,

where $\alpha_{n}$ and $\beta_{n}$ are real coefficients with canonical mass dimension $M^{-2(n+1)}$. Let

$$
\begin{aligned}
& \left\{m_{(2), 1}^{2}, m_{(2), 2}^{2}, \ldots, m_{(2), \tilde{q}+1}^{2}\right\} \quad \text { and } \\
& \left\{m_{(0), 1}^{2}, m_{(0), 2}^{2}, \ldots, m_{(0), \tilde{N}+1}^{2}\right\}
\end{aligned}
$$

be, respectively, the set of real roots of the polynomial functions $Q_{2}\left(k^{2}\right)$ and $Q_{0}\left(k^{2}\right)$, while

$$
\begin{aligned}
& \left\{\eta_{(2), 1}^{2}, \eta_{(2), 1}^{* 2}, \ldots, \eta_{(2), r}^{2}, \eta_{(2), r}^{* 2}\right\} \quad \text { and } \\
& \left\{\eta_{(0), 1}^{2}, \eta_{(0), 1}^{* 2}, \ldots, \eta_{(0), s}^{2}, \eta_{(0), s}^{* 2}\right\}
\end{aligned}
$$

denote, respectively, the sets of complex roots of the polynomials $Q_{2}\left(k^{2}\right)$ and $Q_{0}\left(k^{2}\right)$. Therefore, we arrive at the constraints

\footnotetext{
${ }^{5}$ We have considered the de Donder gauge condition in the computation of the free propagator.
} 


$$
q=\tilde{q}+2 r \quad \text { and } \quad \max \{p, q\}=\tilde{N}+2 s \equiv N .
$$

By the factorization theorem for polynomials and partial fraction decomposition one may write (10) as follows:

$$
\begin{aligned}
\left.P_{00,00}(k)\right|_{k_{0}=0}= & -\frac{1}{\sigma}\left(\frac{D-3}{D-2}\right) \frac{1}{\mathbf{k}^{2}}-\frac{1}{\sigma}\left(\frac{D-2}{D-1}\right) \\
& \times \sum_{i=1}^{q+1} \prod_{\substack{j=1 \\
j \neq i}}^{q+1} \frac{\mu_{(2), j}^{2}}{\mu_{(2), j}^{2}-\mu_{(2), i}^{2}} \frac{1}{\mathbf{k}^{2}+\mu_{(2), i}^{2}} \\
& +\frac{1}{\sigma} \frac{1}{(D-1)(D-2)} \\
& \times \sum_{i=1}^{N+1} \prod_{\substack{j=1 \\
j \neq i}}^{N+1} \frac{\mu_{(0), j}^{2}}{\mu_{(0), j}^{2}-\mu_{(0), i}^{2}} \frac{1}{\mathbf{k}^{2}+\mu_{(0), i}^{2}},
\end{aligned}
$$

where we have defined

$$
\mu_{(2), i}= \begin{cases}m_{(2), i}, & i=1, \ldots, \tilde{q}+1, \\ \eta_{(2), i}, & i=\tilde{q}+2, \ldots, \tilde{q}+r+1, \\ \eta_{(2), i}^{*}, & i=\tilde{q}+r+2, \ldots, \tilde{q}+2 r+1,\end{cases}
$$

and

$\mu_{(0), i}= \begin{cases}m_{(0), i}, & i=1, \ldots, \tilde{N}+1, \\ \eta_{(0), i}, & i=\tilde{N}+2, \ldots, \tilde{N}+s+1, \\ \eta_{(0), i}^{*}, & i=\tilde{N}+s+2, \ldots, \tilde{N}+2 s+1 .\end{cases}$

Substituting (18) into (9) and taking into account the integrals

$\int \frac{d^{D-1} \mathbf{k}}{(2 \pi)^{D-1}} \frac{e^{i \mathbf{k} \cdot \mathbf{r}}}{\mathbf{k}^{2}+\mu^{2}}=\frac{1}{(2 \pi)^{\frac{D-1}{2}}}\left(\frac{\mu}{r}\right)^{\frac{D-3}{2}} K_{\frac{D-3}{2}}(\mu r), \quad$ for $D \geq 3$

$\int \frac{d^{D-1} \mathbf{k}}{(2 \pi)^{D-1}} \frac{e^{i \mathbf{k} \cdot \mathbf{r}}}{\mathbf{k}^{2}}=\frac{1}{(2 \pi)^{\frac{D-1}{2}}} \frac{2^{\frac{D-5}{2}}}{r^{D-3}} \Gamma\left(\frac{D-3}{2}\right), \quad$ for $D \geq 4$,

we find that the $D$-dimensional gravitational potential energy is given by (for $D \geq 4$ )

$$
\begin{aligned}
E_{D}(r)= & -\frac{\kappa^{2} M_{1} M_{2}}{4 \sigma(2 \pi)^{\frac{D-1}{2}}}\left\{\left(\frac{D-3}{D-2}\right) 2^{\frac{D-5}{2}} \Gamma\left(\frac{D-3}{2}\right) \frac{1}{r^{D-3}}\right. \\
& -\left(\frac{D-2}{D-1}\right) \sum_{\substack { i=1 \\
\begin{subarray}{c}{j+1 \\
j \neq i{ i = 1 \\
\begin{subarray} { c } { j + 1 \\
j \neq i } }\end{subarray}}^{q+1} \frac{\mu_{(2), j}^{2}}{\mu_{(2), j}^{2}-\mu_{(2), i}^{2}}\left(\frac{\mu_{(2), i}}{r}\right)^{\frac{D-3}{2}} \\
& \times K_{\frac{D-3}{2}}\left(\mu_{(2), i} r\right)+\frac{1}{(D-1)(D-2)} \\
& \left.\times \sum_{i=1}^{N+1} \prod_{\substack{j=1 \\
j \neq i}}^{N+1} \frac{\mu_{(0), j}^{2}}{\mu_{(0), j}^{2}-\mu_{(0), i}^{2}}\left(\frac{\mu_{(0), i}}{r}\right)^{\frac{D-3}{2}} K_{\frac{D-3}{2}}\left(\mu_{(0), i} r\right)\right\} .
\end{aligned}
$$

Similarly, for $D=3$ the interparticle gravitational potential energy is determined to be

$$
\begin{aligned}
E_{3}(r)= & \frac{\kappa^{2} M_{1} M_{2}}{8 \sigma(2 \pi)}\left\{\sum_{i=1}^{q+1} \prod_{\substack{j=1 \\
j \neq i}}^{q+1} \frac{\mu_{(2), j}^{2}}{\mu_{(2), j}^{2}-\mu_{(2), i}^{2}} K_{0}\left(m_{(2), i} r\right)\right. \\
& \left.-\sum_{i=1}^{N+1} \prod_{\substack{j=1 \\
j \neq i}}^{N+1} \frac{\mu_{(0), j}^{2}}{\mu_{(0), j}^{2}-\mu_{(0), i}^{2}} K_{0}\left(m_{(0), i} r\right)\right\} .
\end{aligned}
$$

We remark that the expression for the nonrelativistic potential energy of the same class of higher-derivative models that we are considering here was already computed in the particular case of $D=4[25,26]$. Also, we note that Ref. [34] presents the nonrelativistic potential energy obtained for higher-order gravities containing the Ricci scalar sector. It is important to emphasize that our results agree with both of them when proper limits are taken.

Our next step will be to analyze the behavior of this gravitational potential energy for small distances. Defining $\nu=\frac{D-3}{2}$, we shall make a distinction between $D$ odd and even, since a modified Bessel function of the second kind $K_{\nu}(x)$ has a Taylor's series expansion which depends if $\nu$ is integer or half-integer.

\section{A. Regularity of the potential energy at the origin for $D$ even}

If the potential energy is defined in a spacetime with even dimensions, we can expand the modified Bessel function of the second kind according to

$$
K_{\nu}(z)=\frac{\pi \csc (\pi \nu)}{2}\left(\sum_{k=0}^{\infty} \frac{1}{\Gamma(k-\nu+1) k !}\left(\frac{z}{2}\right)^{2 k-\nu}-\sum_{k=0}^{\infty} \frac{1}{\Gamma(k+\nu+1) k !}\left(\frac{z}{2}\right)^{2 k+\nu}\right)
$$

and, substituting the above expression in (22), we determine the gravitational potential energy for small distances to be 


$$
\begin{aligned}
E_{D}(r)= & -\frac{\kappa^{2} M_{1} M_{2}}{4 \sigma(2 \pi)^{\nu+1}}\left\{\frac{1}{r^{2 \nu}} \Delta_{\nu}^{\mathrm{even}}(r ; q, N)+\frac{\pi \csc (\pi \nu)}{2^{\nu+1} \Gamma(\nu+1)}\left[\left(\frac{2 \nu+1}{2 \nu+2}\right)\right.\right. \\
& \left.\left.\times \sum_{i=1}^{q+1} \prod_{\substack{j=1 \\
j \neq i}}^{q+1} \frac{\mu_{(2), j}^{2}}{\mu_{(2), j}^{2}-\mu_{(2), i}^{2}} \mu_{(2), i}^{2 \nu}-\frac{1}{(2 \nu+2)(2 \nu+1)} \sum_{i=1}^{N+1} \prod_{\substack{j=1 \\
j \neq i}}^{N+1} \frac{\mu_{(0), j}^{2}}{\mu_{(0), j}^{2}-\mu_{(0), i}^{2}} \mu_{(0), i}^{2 \nu}\right]\right\}+\mathcal{O}(r),
\end{aligned}
$$

where

$$
\begin{aligned}
\Delta_{\nu}^{\mathrm{even}}(r ; q, N)= & \frac{2^{\nu} \Gamma(\nu+1)}{2 \nu+1}-\pi \csc (\pi \nu) \sum_{k=0}^{\nu-\frac{1}{2}} \frac{r^{2 k}}{2^{2 k-\nu+1} \Gamma(k-\nu+1) k !} \\
& \times\left(\frac{2 \nu+1}{2 \nu+2} \sum_{i=1}^{q+1} \prod_{\substack{j=1 \\
j \neq i}}^{q+1} \frac{\mu_{(2), j}^{2}}{\mu_{(2), j}^{2}-\mu_{(2), i}^{2}} \mu_{(2), i}^{2 k}-\frac{1}{(2 \nu+2)(2 \nu+1)} \sum_{i=1}^{N+1} \prod_{\substack{j=1 \\
j \neq i}}^{N+1} \frac{\mu_{(0), j}^{2}}{\mu_{(0), j}^{2}-\mu_{(0), i}^{2}} \mu_{(0), i}^{2 k}\right) .
\end{aligned}
$$

Therefore, the cancellation of Newtonian singularity depends on the behavior of the function $\Delta_{\nu}^{\text {even }}(r ; q, N)$. With the help of a computer algebra system we may verify that this function will be null for all values of $r$ if the following condition is satisfied:

$$
2 q+4-D \geq 0
$$

At first glance this result might seem surprising, after all, we are concluding that the finiteness of the potential near the origin is independent of the scalar curvature squared sector. We shall discuss in Sec. VI the reasons for this being so. It is also worthwhile to note that we could obtain finite results at $r=0$ even if the above condition is not met. However, we would need to adjust the parameters of the Lagrangian.

\section{B. Regularity of the potential energy at the origin for $D$ odd}

For a spacetime with odd dimensions and $D>4$, we can expand the modified Bessel function of the second kind according to

$$
\begin{aligned}
K_{\nu}(z)= & (-1)^{\nu-1} \ln \left(\frac{z}{2}\right) \sum_{k=0}^{\infty} \frac{1}{k !(k+\nu) !}\left(\frac{z}{2}\right)^{\nu+2 k}+\frac{1}{2}\left(\frac{2}{z}\right)^{\nu} \sum_{k=0}^{\nu-1} \frac{(-1)^{k}(\nu-k-1) !}{k !}\left(\frac{z}{2}\right)^{2 k} \\
& +\frac{(-1)^{\nu}}{2} \sum_{k=0}^{\infty} \frac{\psi(k+1)+\psi(k+\nu+1)}{k !(k+\nu) !}\left(\frac{z}{2}\right)^{\nu+2 k},
\end{aligned}
$$

and, substituting the above expression in (22), we found that the gravitational potential energy for small distances is

$$
\begin{aligned}
E_{D}(r)= & -\frac{\kappa^{2} M_{1} M_{2}}{4 \sigma(2 \pi)^{\frac{D-1}{2}}}\left\{\frac{1}{r^{2 \nu}} \Delta_{\nu}^{\text {odd }}(r ; q, N)+\frac{(-1)^{\nu+1}}{2^{\nu+1} \nu !}[(\psi(1)+\psi(\nu+1))\right. \\
& \times\left(\frac{2 \nu+1}{2 \nu+2} \sum_{i=1}^{q+1} \prod_{\substack{j=1 \\
j \neq i}}^{q+1} \frac{\mu_{(2), j}^{2}}{\mu_{(2), j}^{2}-\mu_{(2), i}^{2}} \mu_{(2), i}^{2 \nu}-\frac{1}{(2 \nu+2)(2 \nu+1)} \sum_{i=1}^{N+1} \prod_{\substack{j=1 \\
j \neq i}}^{N+1} \frac{\mu_{(0), j}^{2}}{\mu_{(0), j}^{2}-\mu_{(0), i}^{2}} \mu_{(0), i}^{2 \nu}\right) \\
& -\frac{2 \nu+1}{2 \nu+2} \sum_{i=1}^{q+1} \prod_{\substack{j=1 \\
j \neq i}}^{q+1} \frac{\mu_{(2), j}^{2}}{\mu_{(2), j}^{2}-\mu_{(2), i}^{2}} \mu_{(2), i}^{2 \nu} \ln \left(\mu_{(2), i}^{2}\right) \\
& \left.+\frac{1}{(2 \nu+2)(2 \nu+1)}\left(\sum_{i=1}^{N+1} \prod_{\substack{j=1 \\
j \neq i}}^{N+1} \frac{\mu_{(0), j}^{2}}{\mu_{(0), j}^{2}-\mu_{(0), i}^{2}} \mu_{(0), i}^{2 \nu} \ln \left(\mu_{(0), i}^{2}\right)\right]+\mathcal{O}(r)\right\},
\end{aligned}
$$

where we have defined 


$$
\begin{aligned}
\Delta_{\nu}^{\mathrm{odd}}(r ; q, N)= & \frac{2^{\nu} \Gamma(\nu+1)}{2 \nu+1}-\sum_{k=0}^{\nu-1} \frac{(-1)^{k} 2^{\nu-1}(\nu-k-1) !}{k !}\left(\frac{r}{2}\right)^{2 k} \\
& \times\left(\frac{2 \nu+1}{2 \nu+2} \sum_{i=1}^{q+1} \prod_{\substack{j=1 \\
j \neq i}}^{q+1} \frac{\mu_{(2), j}^{2}}{\mu_{(2), j}^{2}-\mu_{(2), i}^{2}} \mu_{(2), i}^{2 k}-\frac{1}{(2 \nu+2)(2 \nu+1)} \sum_{i=1}^{N+1} \prod_{\substack{j=1 \\
j \neq i}}^{N+1} \frac{\mu_{(0), j}^{2}}{\mu_{(0), j}^{2}-\mu_{(0), i}^{2}} \mu_{(0), i}^{2 k}\right) \\
& +\frac{(-1)^{\nu}}{2^{\nu} \nu !} r^{2 \nu} \ln \left(\frac{r}{2}\right)\left(\frac{2 \nu+1}{2 \nu+2} \sum_{i=1}^{q+1} \prod_{\substack{j=1 \\
j \neq i}}^{q+1} \frac{\mu_{(2), j}^{2}}{\mu_{(2), j}^{2}-\mu_{(2), i}^{2}} \mu_{(2), i}^{2 \nu}\right. \\
& \left.-\frac{1}{(2 \nu+2)(2 \nu+1)} \sum_{i=1}^{N+1} \prod_{\substack{j=1 \\
j \neq i}}^{N+1} \frac{\mu_{(0), j}^{2}}{\mu_{(0), j}^{2}-\mu_{(0), i}^{2}} \mu_{(0), i}^{2 \nu}\right) .
\end{aligned}
$$

Analogously to the above section, the finiteness of potential energy at $r=0$ will depend on the condition $\Delta_{\nu}^{\text {odd }}(r ; q, N)=0$ for every value of the coordinate $r$. With the help of a computer algebra system we can verify that a sufficient condition to get $\Delta_{\nu}^{\text {odd }}(r ; q, N)=0$ is given by

$$
2 q+3-D \geq 0
$$

and the same conclusion obtained in the above section is valid.

\section{Regularity of the potential energy at the origin for $D=3$}

Now let us investigate the three-dimensional case. Considering the expansion

$$
K_{0}(z)=-\ln \left(\frac{z}{2}\right)-\gamma+\mathcal{O}\left(z^{2}\right)
$$

where $\gamma$ is the Euler-Mascheroni constant, it is straightforward to see that, for $D=3$, the gravitational potential energy for small distances is given by

$$
\begin{aligned}
E_{3}(r)= & -\frac{\kappa^{2} M_{1} M_{2}}{16 \pi \sigma}\left\{\left[\sum_{i=1}^{q+1} \prod_{\substack{j=1 \\
j \neq i}}^{q+1} \frac{\mu_{(2), j}^{2}}{\mu_{(2), j}^{2}-\mu_{(2), i}^{2}}-\sum_{i=1}^{N+1} \prod_{\substack{j=1 \\
j \neq i}}^{N+1} \frac{\mu_{(0), j}^{2}}{\mu_{(0), j}^{2}-\mu_{(0), i}^{2}}\right]\left(\ln \left(\frac{r}{2}\right)+\gamma\right)\right. \\
& \left.+\sum_{i=1}^{q+1} \prod_{\substack{j=1 \\
j \neq i}}^{q+1} \frac{\mu_{(2), j}^{2}}{\mu_{(2), j}^{2}-\mu_{(2), i}^{2}} \ln \mu_{(2), i}-\sum_{i=1}^{N+1} \prod_{\substack{j=1 \\
j \neq i}}^{N+1} \frac{\mu_{(0), j}^{2}}{\mu_{(0), j}^{2}-\mu_{(0), i}^{2}} \ln \mu_{(0), i}+\mathcal{O}(r)\right\} .
\end{aligned}
$$

In such a case we can use the identity ${ }^{6}$

$$
\sum_{i=1}^{n+1} \prod_{\substack{j=1 \\ j \neq i}}^{n+1} \frac{a_{j}}{a_{j}-a_{i}}=1
$$

valid for any set of complex numbers $\left\{a_{1}, a_{2}, \ldots, a_{n+1}\right\}$ with $n \geq 0$, in order to verify the following equations:

$$
\sum_{i=1}^{q+1} \prod_{\substack{j=1 \\ j \neq i}}^{q+1} \frac{\mu_{(2), j}^{2}}{\mu_{(2), j}^{2}-\mu_{(2), i}^{2}}=1 \quad \text { and } \quad \sum_{i=1}^{N+1} \prod_{\substack{j=1 \\ j \neq i}}^{N+1} \frac{\mu_{(0), j}^{2}}{\mu_{(0), j}^{2}-\mu_{(0), i}^{2}}=1
$$

for $q \geq 0$ (note that $q \geq 0$ automatically implies $N \geq 0$ ). Using the result above we can recast the interparticle potential energy for small distances as follows:

$$
E_{3}(r)=-\frac{\kappa^{2} M_{1} M_{2}}{16 \pi \sigma}\left\{\sum_{i=1}^{q+1} \prod_{\substack{j=1 \\ j \neq i}}^{q+1} \frac{\mu_{(2), j}^{2} \ln \mu_{(2), i}}{\mu_{(2), j}^{2}-\mu_{(2), i}^{2}}-\sum_{i=1}^{N+1} \prod_{\substack{j=1 \\ j \neq i}}^{N+1} \frac{\mu_{(0), j}^{2} \ln \mu_{(0), i}}{\mu_{(0), j}^{2}-\mu_{(0), i}^{2}}+\mathcal{O}(r)\right\} .
$$

\footnotetext{
${ }^{6}$ For a rigorous proof of this identity we refer to [25].
} 
Therefore, a sufficient condition for cancellation of the Newtonian singularity in three-dimensional higher-derivative gravity is given by $q \geq 0$. In other words, this condition tells us that the existence of the Ricci squared sector is sufficient for the cancellation of Newtonian singularities.

Finally, it is important to emphasize that the above discussion is valid only for higher-derivative theories with unrelated parameters, otherwise the conditions for the cancellation of Newtonian singularities are not sufficient. The so-called new massive gravity (NMG) [35] is a good example of this statement. In this theory the parameters are constrained by $3 \alpha_{0}+8 \beta_{0}=0$ (and $\sigma=-1$ ) and the interparticle potential energy for the NMG may be written as

$$
E_{\mathrm{NMG}}(r)=-\frac{\kappa^{2} M_{1} M_{2}}{16 \pi} K_{0}\left(m_{(2), 1} r\right),
$$

where $m_{(2), 1}^{2}=4 / \beta_{0}$. Taking into account the expansion of Bessel function for small arguments we arrive at the result

$E_{\mathrm{NMG}}(r)=\frac{\kappa^{2} M_{1} M_{2}}{16 \pi}\left[\gamma+\ln \left(\frac{m_{(2), 1} r}{2}\right)\right]+\mathcal{O}(r)$.

Therefore, we have found a divergent potential energy at $r=0$. In Sec. VI we will discuss the mechanism for cancellation of Newtonian singularities which will clearly show why this phenomenon does not occur in NMG theory.
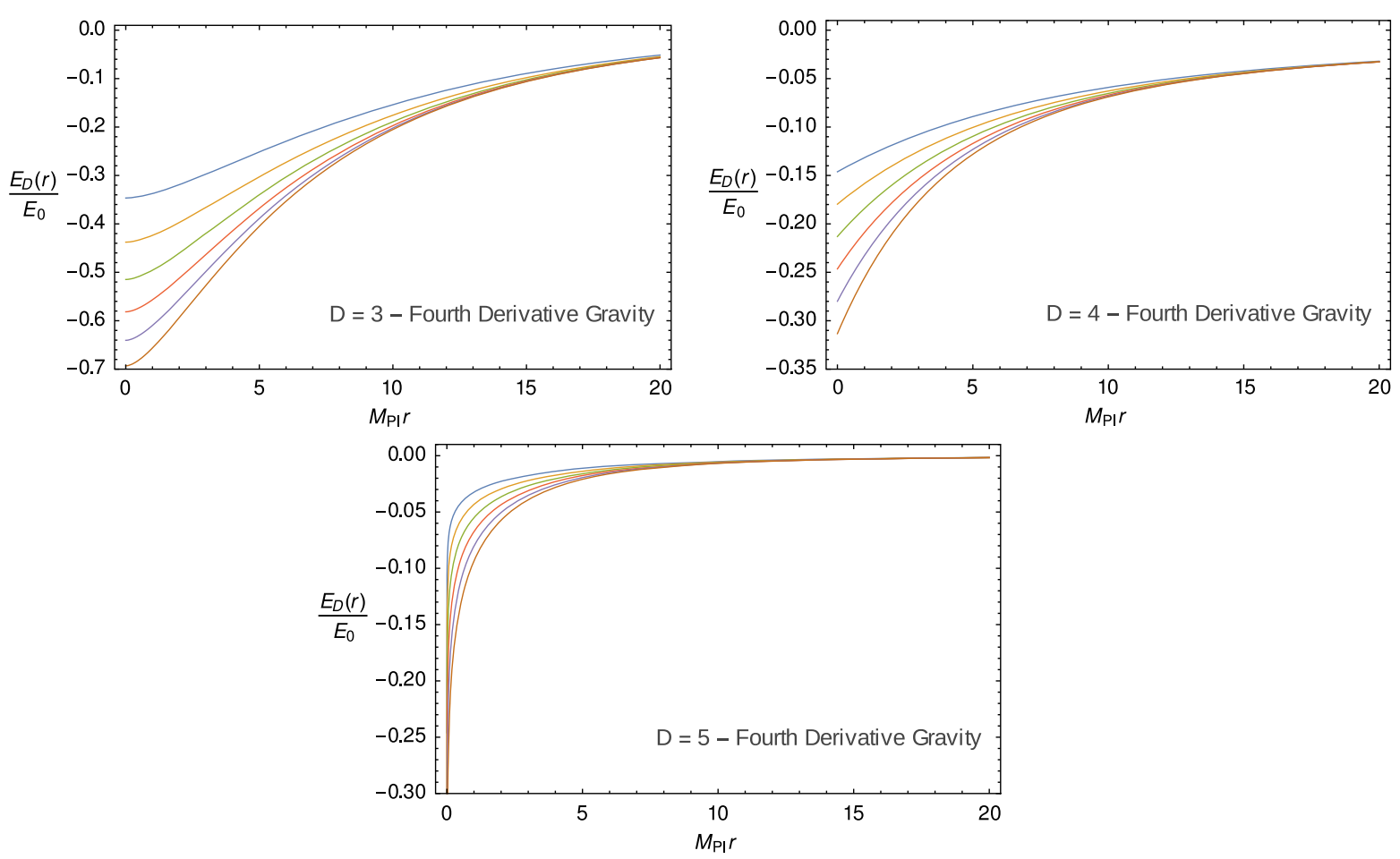

FIG. 1. Nonrelativistic gravitational potential associated with fourth-derivative models.

\section{Plotting results}

In order to complete the discussion of the previous sections let us analyze the graphical behavior of the nonrelativistic potential energy calculated above. Since we want to deal with plots associated with dimensionless quantities, let us define a reference energy (mass) scale given by

$$
E_{0}=\frac{4 M_{1} M_{2}}{(2 \pi)^{\frac{D-3}{2}} M_{\mathrm{Pl}}}
$$

where $M_{\mathrm{Pl}}$ is the planck mass. In this section we will plot $E_{D}(r) / E_{0}$ as a function of $M_{\mathrm{Pl}} r$.

In Fig. 1 we plot those results associated with fourthderivative models for three different values of the spacetime dimension $(D=3,4$ and 5). In all cases we have considered several choices of the mass parameters $m_{(0), 1}$ and $m_{(2), 1}$ (close to the Planck mass $M_{\mathrm{Pl}}$ ), however, their specific values are not relevant for this qualitative analysis. Also, it has been chosen $\sigma=1$ in order to ensure the attractive behavior of the gravitational interaction. As one can see, both for $D=3$ and $D=4$ the nonrelativistic potential energy turns out to be finite at $r=0$. On the other hand, the case $D=5$ exhibits the so-called Newtonian singularity at $r=0$. It is important to emphasize that all these cases are in agreement with the aforementioned sufficient condition for the absence of Newtonian singularities. In addition, we remark that we are not plotting results for $D>5$, since their qualitative behaviors are the same as $D=5$. 


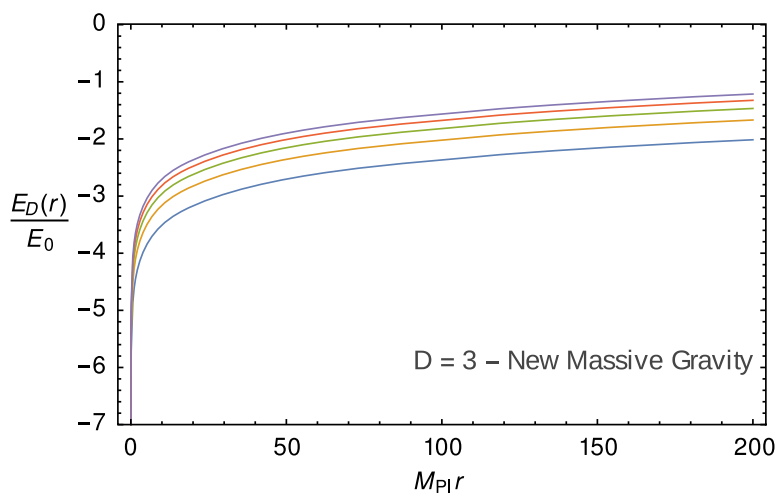

FIG. 2. Nonrelativistic gravitational potential energy for the case of "new massive gravity."
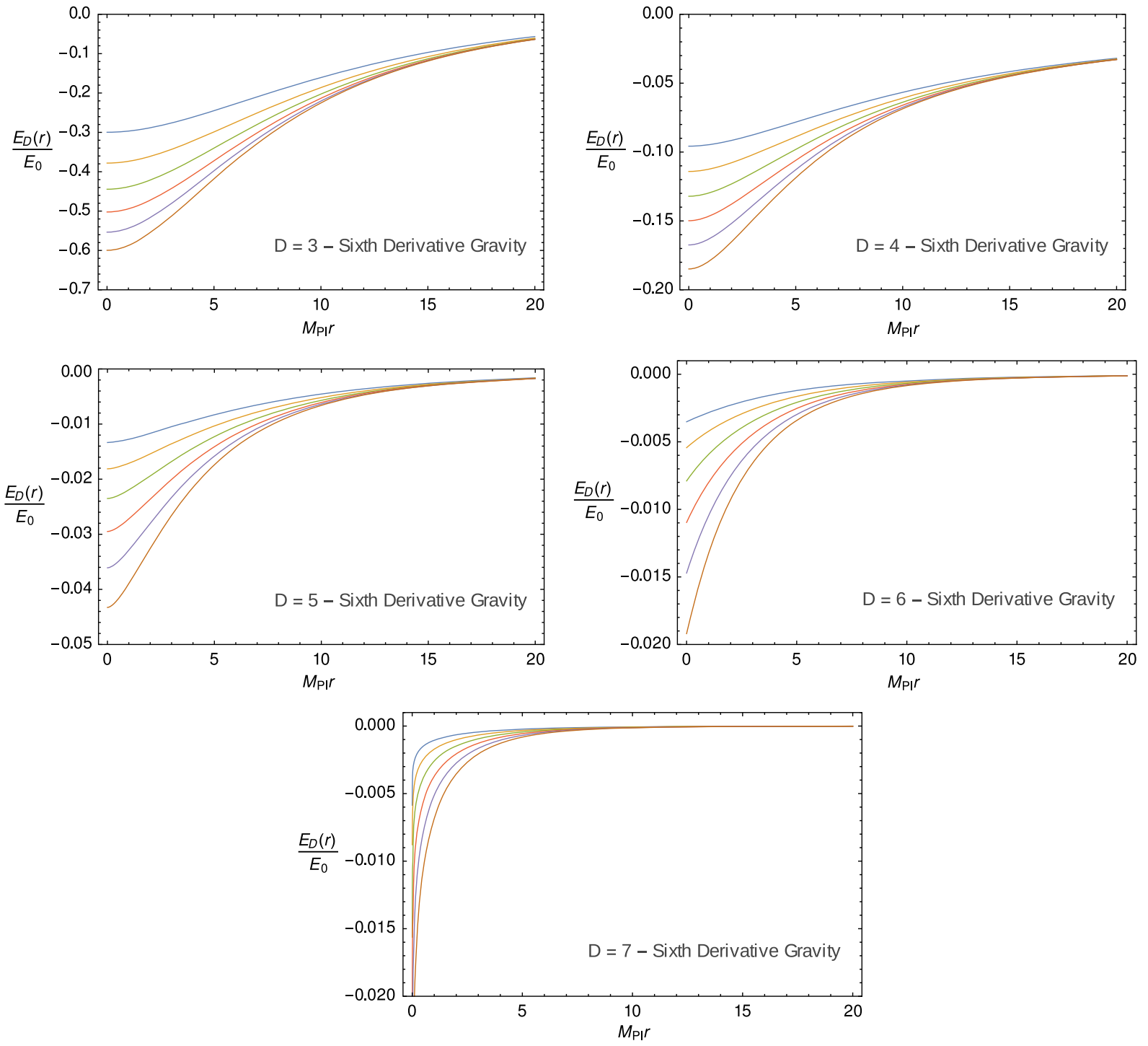

FIG. 3. Nonrelativistic gravitational potential associated with sixth-derivative models. 
$(D=3,4,5,6$ and 7). In all cases we have considered several choices for the massive parameters associated with higher-derivative terms, however, once again, their specific values are irrelevant for the general qualitative behavior discussed here. Remarkably, the nonrelativistic potential corresponding to $D=3,4,5$ and 6 turns out to be finite at $r=0$, while the case $D=7$ exhibits the so-called Newtonian singularity. Furthermore, the graphical behavior obtained with $D>7$ presents the same qualitative features as those corresponding to $D=7$. In all cases, those results presented are in agreement with the sufficient condition for the cancellation of Newtonian singularities obtained before.

Although we have considered only the case of fourthand sixth-derivative models, the analysis performed in this section is exhaustive and can be extended to higher-order gravities. In all cases the graphical behavior should reflect the conclusions obtained by analytical means in the previous section.

\section{UV PROPERTIES AND RENORMALIZABILITY}

As is well known, the motivation for considering higher derivative theories of quantum gravity as possible candidates for quantum gravity models is their good UV properties. As we have already mentioned, Stelle proved that fourth-derivative gravity theories are renormalizable (in four dimensions) to all orders in perturbation theory [6]. Furthermore, theories containing sixth or higher derivatives may be formulated as superrenormalizable [8-10]. For completeness' sake in this section we shall analyze the UV properties of the general class of $D$-dimensional higherderivative theories described by the action (1).

The UV aspects of renormalizability of similar models have been studied in Refs. [6-9]. In addition, as it was stressed out in Ref. [36], power-counting criteria is just a hint for renormalizability and it should be checked with more involved methods, since there could be divergent subgraphs. We note, however, that even if a power-counting renormalizable theory without Newtonian singularity turns out to be nonrenormalizable, the conjecture discussed in this paper is still not falsified. The conjecture states that a finite potential at $r=0$ is a necessary condition for renormalizability, not a sufficient one.

Having said that, we proceed to construct the powercounting criteria for renormalizability of the general class of $D$-dimensional higher-derivative theories described by the action (1). The propagators and vertices associated with this general class of higher-derivative theories have the following UV behavior ${ }^{7}$

$$
\text { Propagators } \sim \frac{1}{k^{2 q+4}} \text { and } \text { Vertices } \sim k^{2 N+4} .
$$

\footnotetext{
${ }^{7}$ Recall that the parameters $q$ and $N$ were introduced in the previous section and are related to the number of derivatives contained in the action.
}

As a consequence, for an arbitrary Feynman diagram the UV behavior of the loop integrations is given by

$$
\mathcal{I}_{\mathrm{UV}}^{\text {Loops }} \sim \int\left(d^{D} k\right)^{L} \frac{\left(k^{2 N+4}\right)^{V}}{\left(k^{2 q+4}\right)^{I}},
$$

where $I$ is the number of internal lines, $L$ stands for the number of loops and $V$ denotes the vertex number. Therefore the superficial degree of divergence associated with the integral may be cast as follows:

$$
\delta=D L+(2 N+4) V-(2 q+4) I .
$$

Now, bearing in mind the topological relations

$$
L-1=I-V,
$$

and

$$
2 I+E=\sum_{n=3}^{\infty} n V_{n}
$$

where $E$ is the number of external lines and $V_{n}$ denotes the number of vertices connecting $n$-lines, we may recast the superficial degree of divergence as

$$
\begin{aligned}
\delta= & D-\sum_{n=3}^{\infty}\left[\frac{n-2}{2}(2 q+4-D)-2 \lambda\right] V_{n} \\
& +\left(\frac{2 q+4-D}{2}\right) E,
\end{aligned}
$$

where the parameter $\lambda$ is given by

$$
\lambda= \begin{cases}p-q, & \text { if } q<p, \\ 0, & \text { if } q \geq p .\end{cases}
$$

As is well known, the power-counting criteria for renormalizability requires that the superficial degree of divergence cannot depend on the number of vertices, therefore, we arrive at the following conditions for power-counting renormalizability:

$$
2 q+4-D=0 \quad \text { and } \quad \lambda=0 .
$$

The first condition relates the number of derivatives in the Ricci-squared sector to the dimension of spacetime. Consequently, in odd dimensions we cannot have powercounting renormalizability since in this case it would be required fractional powers of the d'Alembertian operator. The second condition implies essentially that $q \geq p$. This inequality tells us that the number of derivatives in the scalar curvature squared sector should not be greater than the number of derivatives in the Ricci squared sector. 
Furthermore, the theory can also be formulated as powercounting superrenormalizable. In fact, the condition for superrenormalizability demands that the superficial degree of divergence decreases with the number of vertices. Taking this condition into account, along with (42), we arrive at the strong inequality,

$$
2 q+4-D \geq 4 \lambda
$$

As in the previous case, this inequality relates the number of derivatives in the Ricci squared sector to the dimensionality of spacetime. In addition, if $p>q$ it determines a lower bound on the parameter $q$ in terms of the spacetime dimension and the number of derivatives in the scalar curvature squared sector.

It is important to call attention to the fact that the discussion above relies upon the assumption that the Lagrangian parameters, $\alpha$ 's and $\beta$ 's, are unrelated. In fact, if there existed a link between them, the power counting would be probably slightly changed. To illustrate this, we discuss the so-called new massive gravity (NMG) [35]. This theory is characterized by the choice $F_{1}(\square)=\alpha_{0}$ and $F_{2}(\square)=\beta_{0}$ with the additional constraint

$$
8 \alpha_{0}+3 \beta_{0}=0 .
$$

In order to made the theory ghost-free we have to consider the "wrong" sign in the Einstein-Hilbert sector, i.e., $\sigma=-1$, so that the NMG action becomes

$S_{\mathrm{NMG}}=\int d^{3} x \sqrt{|g|}\left(-\frac{2}{\kappa^{2}} R+\frac{\beta_{0}}{2 \kappa^{2}}\left(R_{\mu \nu}^{2}-\frac{3}{8} R^{2}\right)\right)$.

At first sight, applying the power-counting condition above, NMG should be apparently classified as superrenormalizable. If this was true, NMG would be an example of a ghost-free and superrenormalizable model; however, this is not the case. Indeed, the constraint $3 \alpha_{0}+$ $8 \beta_{0}$ must be considered in such a way that the UV behavior of the tree-level propagator is given by $\sim 1 / k^{2}$. Taking this into account, the correct power counting for the NMG is given by

$$
\delta_{\mathrm{NMG}}=3-\frac{1}{2} E+\frac{1}{2} \sum_{n=3}^{\infty}(n+2) V_{n} \text {. }
$$

As one can see, the superficial degree of divergence increases with the number of vertices and, as a consequence, the theory is power-counting nonrenormalizable. Furthermore, it is important to emphasize that a complete proof of the nonrenormalizability of the NMG was performed in Ref. [37].

\section{PARTICLE SPECTRA AND TREE-LEVEL UNITARITY}

One of the most intriguing problems concerning the formulation of quantum gravity considered as a theory of fluctuations around the Minkowski spacetime is the incompatibility between unitarity and renormalizability. In what follows we shall investigate tree-level unitarity. As usual, this study can be done in terms of the pole structure of the saturated propagator given by

$$
S P(k)=i T^{* \mu \nu}(k) \mathcal{D}_{\mu \nu, \alpha \beta}(k) T^{\alpha \beta}(k),
$$

where $T^{\mu \nu}$ stands for an external conserved current. Using Eq. (10), we arrive at the result

$$
\begin{aligned}
S P(k)= & \frac{i}{\sigma k^{2} Q_{2}\left(k^{2}\right)}\left(T_{\mu \nu}^{*} T^{\mu \nu}-\frac{1}{D-1}|T|^{2}\right) \\
& -\frac{i}{\sigma k^{2} Q_{0}\left(k^{2}\right)} \frac{|T|^{2}}{(D-1)(D-2)} .
\end{aligned}
$$

Considering that the form factors are given by (14), by the factorization theorem for polynomials and partial fraction decomposition we arrive at the result

$$
\begin{aligned}
S P(k)= & \frac{i}{\sigma k^{2}}\left(T_{\mu \nu}^{*} T^{\mu \nu}-\frac{1}{D-2}|T|^{2}\right) \\
& +\frac{|T|^{2}}{\sigma(D-1)(D-2)}\left\{\sum_{i=1}^{\tilde{N}+1} \frac{i \xi_{(0), i}}{k^{2}-m_{(0), i}^{2}}+\sum_{i=1}^{s} \frac{i \zeta_{(0), i}}{k^{2}-\eta_{(0), i}^{2}}+\sum_{i=1}^{s} \frac{i \zeta_{(0), i}^{*}}{k^{2}-\eta_{(0), i}^{* 2}}\right\} \\
& -\frac{1}{\sigma}\left(T_{\mu \nu}^{*} T^{\mu \nu}-\frac{1}{D-1}|T|^{2}\right)\left\{\sum_{i=1}^{\tilde{q}+1} \frac{i \xi_{(2), i}}{k^{2}-m_{(2), i}^{2}}+\sum_{i=1}^{r} \frac{i \zeta_{(2), i}}{k^{2}-\eta_{(2), i}^{2}}+\sum_{i=1}^{r} \frac{i \zeta_{(2), i}^{*}}{k^{2}-\eta_{(2), i}^{* 2}}\right\},
\end{aligned}
$$

where the mass parameters are defined again by the roots of (11) and (12) and, additionally, we have defined 
$\zeta_{(0), i}=\prod_{\substack{j=1 \\ j \neq i}}^{s} \frac{\eta_{(0), j}^{2}}{\eta_{(0), j}^{2}-\eta_{(0), i}^{2}} \prod_{j=1}^{s} \frac{\eta_{(0), j}^{* 2}}{\eta_{(0), j}^{* 2}-\eta_{(0), i}^{2}} \prod_{j=1}^{\tilde{N}+1} \frac{m_{(0), j}^{2}}{m_{(0), j}^{2}-\eta_{(0), i}^{2}}, \quad \operatorname{Im}\left[\operatorname{Res} S P\left(k^{2}=\eta_{(0), i}^{2}\right)\right]=\left.\frac{\sigma^{-1} \operatorname{Im}\left(\zeta_{(0), i}\right)}{(D-1)(D-2)}|T|^{2}\right|_{k^{2}=\eta_{(0), i}^{2}}$,

$\xi_{(0), i}=\prod_{j=1}^{s} \frac{\eta_{(0), j}^{2}}{\eta_{(0), j}^{2}-m_{(0), i}^{2}} \prod_{j=1}^{s} \frac{\eta_{(0), j}^{* 2}}{\eta_{(0), j}^{* 2}-m_{(0), i}^{2}} \prod_{\substack{j=1 \\ j \neq i}}^{\tilde{N}+1} \frac{m_{(0), j}^{2}}{m_{(0), j}^{2}-m_{(0), i}^{2}}$,

$\zeta_{(2), i}=\prod_{\substack{j=1 \\ j \neq i}}^{r} \frac{\eta_{(2), j}^{2}}{\eta_{(2), j}^{2}-\eta_{(2), i}^{2}} \prod_{j=1}^{r} \frac{\eta_{(2), j}^{* 2}}{\eta_{(2), j}^{* 2}-\eta_{(2), i}^{2}} \prod_{j=1}^{\tilde{q}+1} \frac{m_{(2), j}^{2}}{m_{(2), j}^{2}-\eta_{(2), i}^{2}}$,

$\xi_{(2), i}=\prod_{j=1}^{r} \frac{\eta_{(2), j}^{2}}{\eta_{(2), j}^{2}-m_{(2), i}^{2}} \prod_{j=1}^{r} \frac{\eta_{(2), j}^{* 2}}{\eta_{(2), j}^{* 2}-m_{(2), i}^{2}} \prod_{\substack{j=1 \\ j \neq i}}^{\tilde{q}+1} \frac{m_{(2), j}^{2}}{m_{(2), j}^{2}-m_{(2), i}^{2}}$.

As usual, in order to investigate whether the theory exhibits ghosts in its spectrum, we compute the imaginary part of residues of the saturated propagator. Using Eq. (51) we find

$\operatorname{Im}\left[\operatorname{Res} S P\left(k^{2}=0\right)\right]=\left.\sigma^{-1}\left(T_{\mu \nu}^{*} T^{\mu \nu}-\frac{1}{D-2}|T|^{2}\right)\right|_{k^{2}=0}$,

$\operatorname{Im}\left[\operatorname{Res} S P\left(k^{2}=m_{(2), i}^{2}\right)\right]=-\sigma^{-1} \xi_{(2), i}$

$$
\times\left.\left(T_{\mu \nu}^{*} T^{\mu \nu}-\frac{1}{D-1}|T|^{2}\right)\right|_{k^{2}=m_{(2), i}^{2}}
$$

$$
\begin{aligned}
\operatorname{Im}\left[\operatorname{Res} S P\left(k^{2}=\eta_{(2), i}^{2}\right)\right]= & -\sigma^{-1} \operatorname{Im}\left(\zeta_{(2), i}\right) \\
& \times\left.\left(T_{\mu \nu}^{*} T^{\mu \nu}-\frac{1}{D-1}|T|^{2}\right)\right|_{k^{2}=\eta_{(2), i}^{2}},
\end{aligned}
$$

$$
\begin{aligned}
\operatorname{Im}\left[\operatorname{Res} S P\left(k^{2}=\eta_{(2), i}^{* 2}\right)\right]= & -\sigma^{-1} \operatorname{Im}\left(\zeta_{(2), i}^{*}\right) \\
& \times\left.\left(T_{\mu \nu}^{*} T^{\mu \nu}-\frac{1}{D-1}|T|^{2}\right)\right|_{k^{2}=\eta_{(2), i}^{* 2}},
\end{aligned}
$$

$\operatorname{Im}\left[\operatorname{Res} S P\left(k^{2}=m_{(0), i}^{2}\right)\right]=\left.\frac{\sigma^{-1} \xi_{(0), i}}{(D-1)(D-2)}|T|^{2}\right|_{k^{2}=m_{(0), i}^{2}}$,
$\operatorname{Im}\left[\operatorname{Res} S P\left(k^{2}=\eta_{(0), i}^{* 2}\right)\right]=\left.\frac{\sigma^{-1} \operatorname{Im}\left(\zeta_{(0), i}^{*}\right)}{(D-1)(D-2)}|T|^{2}\right|_{k^{2}=\eta_{(0), i}^{* 2}}$.

Assuming that the real masses obey the hierarchy ${ }^{8}$

$$
\begin{aligned}
& m_{(2), 1}^{2}<m_{(2), 2}^{2}<\cdots<m_{(2), \tilde{q}+1}^{2} \quad \text { and } \\
& m_{(0), 1}^{2}<m_{(0), 2}^{2}<\cdots<m_{(0), \tilde{N}+1}^{2},
\end{aligned}
$$

we arrive at the conclusion

$\xi_{(2), i}>0, \quad$ if $i=$ odd,
$\xi_{(2), i}<0, \quad$ if $i=$ even, and $\quad \begin{array}{ll}\xi_{(0), i}>0, & \text { if } i=\text { odd, } \\ \xi_{(0), i}<0, & \text { if } i=\text { even. }\end{array}$

From now on, we divide our analysis in two cases: $D \geq 4$ and $D=3$.

\section{A. Case $I-D \geq 4$}

We first consider the case where $D \geq 4$. Taking into account the set of inequalities (valid for $D \geq 4$ )

$$
\left.\left(T_{\mu \nu}^{*} T^{\mu \nu}-\frac{1}{D-2}|T|^{2}\right)\right|_{k^{2}=0}>0,
$$

and

$$
\left.\left(T_{\mu \nu}^{*} T^{\mu \nu}-\frac{1}{D-1}|T|^{2}\right)\right|_{k^{2}=\mu^{2}}>0, \quad \mu^{2}=m_{(2), i}^{2}, \eta_{(2), i}^{2}, \eta_{(2), i}^{* 2},
$$

we obtain the results

$$
\operatorname{Im}\left[\operatorname{Res} S P\left(k^{2}=0\right)\right] \begin{cases}>0, & \text { for } \sigma=+1 \\ <0, & \text { for } \sigma=-1\end{cases}
$$

$\operatorname{Im}\left[\operatorname{ResSP}\left(k^{2}=m_{(2), i}^{2}\right)\right]\left\{\begin{array}{ll}<0, & \text { for } \sigma=+1 \\ >0, & \text { for } \sigma=-1,\end{array}\right.$ if $i=$ odd,

\footnotetext{
${ }^{8}$ It is important to emphasize that this ordering can always be achieved by relabeling the masses.
} 
$\operatorname{Im}\left[\operatorname{Res} S P\left(k^{2}=m_{(2), i}^{2}\right)\right]\left\{\begin{array}{ll}>0, & \text { for } \sigma=+1 \\ <0, & \text { for } \sigma=-1,\end{array}\right.$ if $i=$ even,

$\operatorname{Im}\left[\operatorname{ResSP}\left(k^{2}=m_{(0), i}^{2}\right)\right]\left\{\begin{array}{ll}>0, & \text { for } \sigma=+1 \\ <0, & \text { for } \sigma=-1,\end{array}\right.$ if $i=$ odd,

$\operatorname{Im}\left[\operatorname{ResSP}\left(k^{2}=m_{(0), i}^{2}\right)\right]\left\{\begin{array}{ll}<0, & \text { for } \sigma=+1 \\ >0, & \text { for } \sigma=-1,\end{array}\right.$ if $i=$ even.

In addition, considering $\operatorname{Im}\left(\zeta_{(I), i}\right)=-\operatorname{Im}\left(\zeta_{(I), i}^{*}\right)$ (where $I=0,2)$, we may conclude that

$$
\begin{aligned}
& \text { if } \operatorname{Im}\left[\operatorname{Res} S P\left(k^{2}=\eta_{(I), i}^{2}\right)\right]>0 \\
& \quad \Rightarrow \operatorname{Im}\left[\operatorname{Res} S P\left(k^{2}=\eta_{(I), i}^{* 2}\right)\right]<0,
\end{aligned}
$$

and

$$
\begin{aligned}
& \text { if } \operatorname{Im}\left[\operatorname{Res} S P\left(k^{2}=\eta_{(I), i}^{2}\right)\right]<0 \\
& \quad \Rightarrow \operatorname{Im}\left[\operatorname{Res} S P\left(k^{2}=\eta_{(I), i}^{* 2}\right)\right]>0 .
\end{aligned}
$$

Taking into account the set of inequalities above we may list some conclusions:

(i) Although our former discussion applies for both $\sigma=$ +1 and $\sigma=-1$, relation (57) implies $\sigma=+1$, since we expect a physical massless spin-2 particle in the spectrum of the theory corresponding to the usual graviton.

(ii) The usual drama of higher derivative theories persists as long as real poles are present. In this case, the alternating signs of the parameters $\xi_{(2), i}$ and $\xi_{(0), i}$ ensure the existence of at least one ghostlike particle. As usual the existence of such particles in the spectrum may lead to a nonunitary $S$-matrix in the context of perturbation theory.

(iii) Since the complex poles always appear in pairs, one of them being the complex conjugate of the other, we may conclude that for each complex "physical" particle (not a ghostlike state) there will be a complex ghost corresponding to the complex conjugated of the former. Although complex ghosts may appear in the particle spectrum of the theory they may not cause problems with the unitarity of the $S$-matrix. In fact, higher-derivative gravity systems with complex ghosts have been recently studied by Modesto and Shapiro [11,12] and there is hope that this kind of theories may be formulated as unitary in the Lee-Wick sense.
The situation now is clear: as long as higher derivatives are implemented by means of polynomial functions of the d'Alembertian operator the presence of at least one massive ghostlike particle appears to be unavoidable. In fact, there are only three cases of higher-derivative gravity models, constructed with polynomial functions like (14), where the particle spectrum does not exhibit a massive ghostlike state. The first one occurs with the choice $F_{1}(\square)=\alpha_{0}$ and $F_{2}(\square)=0$-in this case the propagator has only two poles, $k^{2}=0$ and $k^{2}=m_{(0), 1}^{2}$ and we conclude that both poles correspond to physical particles, i.e., the theory is ghost-free (at least in tree-level analysis). We remark that, as expected, this theory is classified as nonrenormalizable by the power-counting criteria of Sec. IV. The the other two cases occur in $D=3$ and will be considered in the next section.

\section{B. Case II-D=3:}

Now we consider the three-dimensional case. In this situation the energy-momentum tensor satisfies the relations

$$
\left.\left(T_{\mu \nu}^{*} T^{\mu \nu}-|T|^{2}\right)\right|_{k^{2}=0}=0
$$

and

$$
\left.\left(T_{\mu \nu}^{*} T^{\mu \nu}-\frac{1}{2}|T|^{2}\right)\right|_{k^{2}=\mu^{2}}>0, \quad \mu^{2}=m_{(2), i}^{2}, \eta_{(2), i}^{2}, \eta_{(2), i}^{* 2} .
$$

First of all, Eq. (59a) implies in the result

$$
\operatorname{Im}\left[\operatorname{Res} S P\left(k^{2}=0\right)\right]=0,
$$

which tells us that there is no massless propagating mode in the three-dimensional theory. As a consequence of the last equation we cannot fix the parameter $\sigma$ as we have done in the $D \geq 4$ case. As far as the massive poles (real and complex) are concerned, the inequality (59b) implies that the previous constraints, namely (57b)-(58b), remain valid as well as the comments about massive ghosts in 3D.

As it was mentioned previously, in a three-dimensional spacetime there are two ghost-free higher-derivative theories. The first is obtained by choosing the coefficients in such a way that $F_{1}(\square)=\alpha_{0}$ and $F_{2}(\square)=0$. Consequently, the only particle in the spectrum corresponds to the pole $k^{2}=m_{(0), 1}^{2}$ and the theory is ghost-free, since it can be verified that the residue at this pole is positive.

The other one is the previously discussed new massive gravity (NMG) [35]. In this theory the free propagator exhibits two simple poles at $k^{2}=0$ and $k^{2}=m_{(2), 1}^{2} \equiv$ $4 / \beta_{0}$. Contrary to what happens in dimensions other than 3 , the massless pole does not propagate as a physical particle, since $\left.\operatorname{Res} S P\left(k^{2}=0\right)\right|_{D=3}=0$. Usually the second 
pole $k^{2}=m_{(2), 1}^{2}$ would propagate as a massive ghost, but here the wrong sign of the Einstein-Hilbert terms leads to a positive valued residue:

$$
\begin{aligned}
& \left.\operatorname{Im}\left[\operatorname{Res} S P\left(k^{2}=m_{(2), i}^{2}\right)\right]\right|_{\mathrm{NMG}} \\
& \quad=\left.\left(T_{\mu \nu}^{*} T^{\mu \nu}-\frac{1}{2}|T|^{2}\right)\right|_{k^{2}=m_{(2), 1}^{2}}>0 .
\end{aligned}
$$

Consequently the spectrum of NMG has only a single massive physical particle with spin-2 and there is no ghostlike state in the tree-level propagator.

Last but not least, we would like to draw the reader's attention to Ref. [38], where an interesting study of unitarity higher derivative theories was performed on flat and maximally symmetric spaces.

\section{RELATING RENORMALIZABILITY, UNITARITY AND POTENTIAL ENERGY}

In his seminal paper about renormalizability of higher-derivative quantum gravity [6], Stelle hinted at the possibility of an interesting connection between renormalizability of higher-derivative theories of quantum gravity and the cancellation of Newtonian singularities in the interparticle potential energy. It was later proposed that this connection is a general property of $D$-dimensional higher derivative theories of quantum gravity [21]. Essentially this surmise states that a renormalizable theory of quantum gravity should not present the so-called Newtonian singularity. Recently this conjecture was probed in the case of fourth- and sixth-derivative theories of gravity $[22,23]$. We are now ready to verify this conjecture for a general class of $D$-dimensional higher-derivative theories of quantum gravity described by the action (1) with form factors given by (14).

Let us recall two important results from our last two sections. First of all, investigating the UV properties of the general class of higher-derivative theories under consideration, we found the following necessary conditions for (super)renormalizability:

(i) $2 q+4-D=0 \sim$ power-counting renormalizability;

(ii) $2 q+4-D \geq 4 \lambda \sim$ power-counting superrenormalizability,

where we remind that, for the convenience of the reader, $D$ denotes the dimension of spacetime, the parameter $\lambda$ is defined to be

$$
\lambda= \begin{cases}p-q, & \text { if } q<p, \\ 0, & \text { if } q \geq p,\end{cases}
$$

and $q$ and $p$ denote, respectively, the degree of the polynomial form factor of the Ricci tensor sector and the degree of the polynomial form factor of the Ricci scalar sector [as given by (14)].

Furthermore, after an exhaustive investigation on the behavior of the interparticle potential energy for small distances, we found the sufficient conditions for the cancellation of Newtonian singularities:

(i) $2 q+4-D \geq 0$, for even dimensions;

(ii) $2 q+3-D \geq 0$, for odd dimensions.

Putting all of this information together, it is not difficult to conclude that the necessary conditions for (super)renormalizability automatically imply in the sufficient condition for the cancellation of Newtonian singularities. Summing up:

$$
\begin{gathered}
\text { Power counting (super) renormalizability } \\
\Rightarrow \text { Finite potential energy at } r=0 .
\end{gathered}
$$

This completes our examination of the aforementioned conjecture for the general class of theories under consideration. It should be emphasized that the inverse of this conjecture is not necessarily true, i.e., the cancellation of Newtonian singularities does not imply renormalizability. In fact, as it was pointed out by Giacchini, it is not difficult to construct an example of a higher-derivative model with finite potential energy at $r=0$ and power-counting nonrenormalizable [25].

Finally, let us discuss in passing the role of (non)unitarity on the connection between renormalizability and Newtonian singularities. As we can see from the previous section, ghost-free higher-derivative (local) theories are not compatible with renormalizability. The reason for this point relies on the crucial role played by the ghostlike particles in the improvement of the tree-level propagator. Furthermore, ghosts are also necessary in the cancellation of Newtonian singularities $[25,26]$. For instance, is not difficult to verify that those higher-derivative theories which are ghost-free, have a divergent potential energy at $r=0$.

Indeed, the fact that ghostlike particles are necessary for the cancellation of Newtonian singularities has an interesting explanation. From the classical point of view, ghostlike particles correspond to negative-energy propagation modes (which gives rise to Ostrogradsky instabilities, for instance). Taking into account, from a heuristic point of view, that the interparticle potential energy is given by the sum of individual energies associated with each propagation mode, it is necessary to have parts with opposite signs in order to have some kind of cancellation. Therefore, negative-energy propagation modes are necessary for the cancellation of Newtonian singularities.

The role of ghost-free particles in the mechanism for cancellation of Newtonian singularities was recently explored in the literature. In fact, Modesto and collaborators demonstrated that four-dimensional theories described by (1), with $F_{1}(\square)$ and $F_{2}(\square)$ being polynomial functions with the same degree, i.e., the same number of ghosts and physical particles, the cancellation of Newtonian 
singularities occurs [26]. Later, Giacchini demonstrated the equal number of ghosts and physical particle is not a necessary condition for the cancellation of Newtonian singularities in four-dimensional higher-derivative gravity [25]. The necessary condition is that the particle spectra of the theory should contain at least a massive ghost and a massive physical particle (besides the usual massless graviton). In the case of three-dimensional theories, it is not difficult to adapt Giacchini's demonstration in order to get the same conclusion. However, in the case of spacetime with dimension higher than 4 the situation is more subtle. Although we have no demonstration, the above proposition appears to be valid as well, nevertheless, the minimal number of both massive ghosts and physical particles increases with the dimension of spacetime.

\section{FINAL REMARKS}

In this paper we discussed some aspects concerning the tree-level unitarity, renormalizability properties and their relation to the classical context. Our primary aim in this paper was to prove a conjecture which states that (super) renormalizable theories of quantum gravity do not present the so-called Newtonian singularity in the potential energy. As we have seen, the necessary condition for powercounting (super)renormalizability automatically leads to a sufficient condition for the cancellation of Newtonian singularities. We analyzed the role of ghostlike particles in the mechanism of cancellation of Newtonian singularities as well.

We also performed an exhaustive research of the particle spectra and the presence of ghostlike particles and showed that the appearance of them in higher-derivative theories seems to be unavoidable. However, there are two exceptions to this statement: a special case of $f(R)$-theories and new massive gravity.

We studied also the UV properties of a general class of higher-derivative theories. We found the necessary conditions for power-counting (super)renormalizability, relating the number of derivatives to spacetime dimension. Unfortunately, the models which are tree-level ghost-free turn out to be nonrenormalizable. This fact clearly shows the impossibility of reconciling renormalizability with unitarity in quantum gravity. A possible way to circumvent this problem is to use nonlocal theories of quantum gravity $[15,16]$ or to utilize the Lee-Wick formulation of unitarity $[11,12]$. In two recent papers, Anselmi and Piva developed a new formulation of Lee-Wick theories $[39,40]$ and it certainly deserves some investigation in the context of higher-derivative quantum gravities.

It is worth mentioning that we have not yet found a complete proof of the necessary condition for the cancellation of Newtonian singularities in arbitrary dimensions. Our calculations were made with the help of an algebraic manipulation system; on the other hand, their analytical consequences open a new road for finding a general solution to this issue.

Finally, we call attention to the fact that our discussion regarding the connection between (super)renormalizability and the cancellation of Newtonian singularities is based on the assumption of polynomial (local) form factors. We hope that our conclusions may be generalized for nonlocal theories; however, the mechanism concerning the cancellation of Newtonian singularities depends on the specific form of the functions $F_{1}(\square)$ and $F_{2}(\square)$.

\section{ACKNOWLEDGMENTS}

The authors are very grateful to Conselho Nacional de Desenvolvimento Científico e Tecnológico (CNPq) and Fundação de Amparo à Pesquisa do Estado do Rio de Janeiro (FAPERJ) for their financial support. G. P. B. is thankful to The Abdus Salam International Centre for Theorerical Physics for the hospitality when part of this work was done.
[1] C. Kiefer, Quantum Gravity (Oxford University Press, New York, 2004).

[2] D. Oriti, Approaches to Quantum Gravity: Toward a New Understanding of Space, Time and Matter (Cambridge University Press, New York, 2009).

[3] I. L. Buchbinder, S. D. Odintsov, and I. L. Shapiro, Effective Action in Quantum Gravity (IOP Publishing, Bristol, 1992).

[4] J.F. Donoghue, General relativity as an effective field theory: The leading quantum corrections, Phys. Rev. D 50, 3874 (1994).

[5] R. Percacci, An Introduction to Covariant Quantum Gravity and Asymptotic Safety (World Scientific, Singapore, 2017).
[6] K. S. Stelle, Renormalization of higher-derivative quantum gravity, Phys. Rev. D 16, 953 (1977).

[7] E. T. Tomboulis, Unitarity in Higher Derivative Quantum Gravity, Phys. Rev. Lett. 52, 1173 (1984).

[8] M. Asorey, J. L. López, and I. L. Shapiro, Some remarks on high derivative quantum gravity, Int. J. Mod. Phys. A 12, 5711 (1997).

[9] L. Modesto, Super-renormalizable quantum gravity, Phys. Rev. D 86, 044005 (2012).

[10] L.Modesto, Super-renormalizablemultidimensionalquantum gravity:Theoryandapplications, Astron.Rev.8,4(2013).

[11] L. Modesto, Super-renormalizable or finite Lee-Wick quantum gravity, Nucl. Phys. B909, 584 (2016). 
[12] L. Modesto and I. L. Shapiro, Superrenormalizable quantum gravity with complex ghosts, Phys. Lett. B 755, 279 (2016).

[13] H. Yamamoto, Convergent field theory with complex masses, Prog. Theor. Phys. 42, 707 (1969).

[14] T. Biswas, E. Gerwick, T. Koivisto, and A. Mazumdar, Towards Singularity- and Ghost-Free Theories of Gravity, Phys. Rev. Lett. 108, 031101 (2012).

[15] L. Modesto and L. Rachwal, Super-renormalizable and finite gravitational theories, Nucl. Phys. B889, 228 (2014).

[16] L. Modesto and L. Rachwal, Nonlocal quantum gravity: A review, Int. J. Mod. Phys. D 26, 1730020 (2017).

[17] I. L. Shapiro, Counting ghosts in the "ghost-free" nonlocal gravity, Phys. Lett. B 744, 67 (2015).

[18] T. Biswas, A. Mazumdar, W. Siegel, T. Biswas, E. Gerwick, T. Koivisto, and A. Mazumdar, Bouncing universes in string-inspired gravity, J. Cosmol. Astropart. Phys. 03 (2006) 009.

[19] A. S. Koshelev and A. Mazumdar, Do massive compact objects without event horizon exist in infinite derivative gravity?, Phys. Rev. D 96, 084069 (2017).

[20] S. Talaganis and A. Mazumdar, High-energy scatterings in infinite-derivative field theory and ghost-free gravity, Classical Quantum Gravity 33, 145005 (2016).

[21] A. Accioly, J. Helayel-Neto, E. Scatena, and R. Turcati, Solving the riddle of the incompatibility between renormalizability and unitarity in $N$-dimensional Einstein gravity enlarged by curvature-squared terms, Int. J. Mod. Phys. D 22, 1342015 (2013).

[22] A. Accioly, J. Almeida, G. P. Brito, and G. Correia, Renormalizability in D-dimensional higher-order gravity, Phys. Rev. D 95, 084007 (2017).

[23] A. Accioly, G. Correia, G. P. Brito, J. Almeida, and W. Herdy, Relating renormalizability of D-dimensional higherorder electromagnetic and gravitational models to the classical potential at the origin, Mod. Phys. Lett. A 32, 1750048 (2017).

[24] Y. S. Myung, Renormalizability and Newtonian potential in scale-invariant gravity, arXiv:1708.03451.

[25] B. L. Giacchini, On the cancellation of Newtonian singularities in higher-derivative gravity, Phys. Lett. B 766, 306 (2017).

[26] L. Modesto, T. Paula Netto, and I. L. Shapiro, On Newtonian singularities in higher derivative gravity models, J. High Energy Phys. 04 (2015) 098.
[27] T. Biswas, T. Koivisto, and A. Mazumdar, Nonlocal theories of gravity: The flat space propagator, arXiv:1302.0532.

[28] T. Biswas, A. S. Koshelev, and A. Mazumdar, Consistent higher derivative gravitational theories with stable de Sitter and anti-de Sitter backgrounds, Phys. Rev. D 95, 043533 (2017).

[29] T. Biswas, A. S. Koshelev, and A. Mazumdar, Gravitational theories with stable (anti-)de Sitter backgrounds, Fundam. Theor. Phys. 183, 97 (2016).

[30] T. Biswas, A. Conroy, A. S. Koshelev, and A. Mazumdar, Generalized ghost-free quadratic curvature gravity, Classical Quantum Gravity 31, 159501 (2014).

[31] A. Accioly, J. Helayel-Netto, F. E. Barone, and W. Herdy, Simple prescription for computing the interparticle potencial energy for D-dimensional gravity systems, Classical Quantum Gravity 32, 035021 (2015).

[32] A. Accioly, S. Ragusa, H. Mukai, and E. de Rey Neto, Algorithm for computing the propagator for higher derivative gravity theories, Int. J. Theor. Phys. 39, 1599 (2000).

[33] A. Conroy, A. Mazumdar, S. Talaganis, and A. Teimouri, Nonlocal gravity in D dimensions: Propagators, entropy, and a bouncing cosmology, Phys. Rev. D 92, 124051 (2015).

[34] I. Quandt and H. Schmidt, The Newtonian limit of fourth and higher order gravity, Astron. Nachr. 312, 97 (1991).

[35] E. A. Bergshoeff, O. Hohm, and P. K. Townsend, Massive Gravity in Three Dimensions, Phys. Rev. Lett. 102, 201301 (2009).

[36] S. Talaganis, B. Tirthabir, and A. Mazumdar, Towards understanding the ultraviolet behavior of quantum loops in infinite-derivative theories of gravity, Classical Quantum Gravity 32, 215017 (2015).

[37] K. Muneyuki and N. Ohta, Unitarity versus renormalizability of higher derivative gravity in 3D, Phys. Rev. D 85, 101501 (2012).

[38] N. Ohta, A complete classification of higher derivative gravity in 3D and criticality in 4D, Classical Quantum Gravity 29, 015002 (2012).

[39] D. Anselmi and M. Piva, A new formulation of LeeWick quantum field theory, J. High Energy Phys. 06 (2017) 066.

[40] D. Anselmi and M. Piva, Perturbative unitarity of LeeWick quantum field theory, Phys. Rev. D 96, 045009 (2017). 